\title{
A mixed finite element method based on a biorthogonal system for nearly incompressible elastic problems
}

\author{
Bishnu P. Lamichhane ${ }^{1}$
}

(Received 12 August 2008; revised 6 November 2008)

\begin{abstract}
A Petrov-Galerkin scheme in a saddle point formulation gives rise to a non-symmetric saddle point problem. This article considers a non-symmetric saddle point problem with a penalty parameter. A mixed finite element method for linear elasticity based on a PetrovGalerkin formulation is then analyzed within the framework of the non-symmetric saddle point problem with penalty. Working with a biorthogonal system to discretize the pressure equation, we obtain a robust and efficient numerical scheme for nearly incompressible linear elasticity using linear finite elements. A numerical example demonstrates the robustness of the approach. These results are useful to analyze a Petrov-Galerkin scheme in a saddle point problem.
\end{abstract}

http://anziamj.austms.org.au/ojs/index.php/ANZIAMJ/article/view/1422 gives this article, (c) Austral. Mathematical Soc. 2008. Published November 17, 2008. ISSN 1446-8735. (Print two pages per sheet of paper.) 


\section{Contents}

1 Introduction and abstract setting

C325

2 Finite element discretization

C330

3 Numerical results

C335

References

C336

\section{Introduction and abstract setting}

Low order finite elements based on standard displacement formulation exhibit a locking effect when applied to nearly incompressible problems [1, 2]. A standard remedy for the locking effect is to work with mixed methods introducing some extra variables leading to a saddle point problem [3, 4]. Here we consider a simple mixed formulation in displacement-pressure form [4], and apply a Petrov-Galerkin formulation for the pressure equation. The pressure equation is discretized using different trial and test spaces, where the bases of the trial and test spaces form a biorthogonal system. This results in a diagonal mass matrix for the pressure equation, and it is easy to statically condense out the pressure inverting a diagonal matrix. We proposed this formulation in recent work [5] as an alternative approach to the method based on primal and dual meshes. However, the mathematical analysis presented in that study covers only the case of primal and dual meshes. We note that the method based on primal and dual meshes results in a symmetric saddle point system, whereas the Petrov-Galerkin formulation for the pressure equation yields a non-symmetric saddle point system. The novel idea of this article is to show the well-posedness of this formulation by combining the results on the non-symmetric saddle point problem $[6,7,8]$ and the stability result of mini-element [9]. 
We start with an abstract setting. Let $\mathrm{V}, \mathrm{W}, \mathrm{P}$ and $\mathrm{Q}$ be Hilbert spaces with inner products $(\cdot, \cdot)_{\mathrm{V}},(\cdot, \cdot)_{\mathrm{W}},(\cdot, \cdot)_{\mathrm{P}}$ and $(\cdot, \cdot)_{\mathrm{Q}}$, respectively. Let $\mathrm{a}(\cdot, \cdot)$ : $\mathrm{V} \times \mathrm{W} \rightarrow \mathbb{R}, \mathrm{b}_{1}(\cdot, \cdot): \mathrm{W} \times \mathrm{P} \rightarrow \mathbb{R}, \mathrm{b}_{2}(\cdot, \cdot): \mathrm{V} \times \mathrm{Q} \rightarrow \mathbb{R}$, and $\mathrm{c}(\cdot, \cdot):$ $\mathrm{P} \times \mathrm{Q} \rightarrow \mathbb{R}$ be bilinear forms. We consider a non-symmetric saddle point problem with penalty: given $f \in W^{\prime}$ and $g \in Q^{\prime}$, find $(u, p) \in V \times P$ so that

$$
\begin{array}{ll}
\mathrm{a}(\mathrm{u}, w)+\mathrm{b}_{1}(w, p)=\mathrm{f}(w), & w \in W \\
\mathrm{~b}_{2}(\mathrm{u}, \mathrm{q})-\mathrm{tc}(\mathrm{p}, \mathrm{q})=\mathrm{g}(\mathrm{q}), \quad \mathrm{q} \in \mathrm{Q},
\end{array}
$$

where $t$ is a positive small parameter, and $W^{\prime}$ and $Q^{\prime}$ denote the space of continuous linear functionals on $W$ and $Q$, respectively. We are interested in analyzing the well-posedness of the problem (1) when $t \rightarrow 0$. There are a number of publications devoted to the analysis of this problem in different particular forms; for example, when $b_{1}(\cdot, \cdot)$ and $b_{2}(\cdot, \cdot)$ are the same, and $\mathrm{V}=\mathrm{W}$ and $\mathrm{P}=\mathrm{Q}[3,4]$. The case with $\mathrm{t}=0$ was analyzed by Nicolaides [6] and Bernardi et al. [7], whereas the case with $\mathrm{V}=\mathrm{W}, \mathrm{P}=\mathrm{Q}$ and $\mathrm{t}=1$ was considered by Ciarlet et al. [8]. We show the stability of the problem (1) by combining the ideas presented by Ciarlet et al. [8] and Bernardi et al. [7]. To this end, we assume that the bilinear forms $a(\cdot, \cdot), b_{1}(\cdot, \cdot), b_{2}(\cdot, \cdot)$ and $c(\cdot, \cdot)$ satisfy

$$
\begin{aligned}
|\mathrm{a}(v, w)| & \leq \overline{\mathrm{a}}\|v\|_{V}\|w\|_{W}, \quad v \in \mathrm{V}, w \in \mathrm{W}, \\
\left|\mathrm{b}_{1}(w, p)\right| & \leq \overline{\mathrm{b}}_{1}\|w\|_{w}\left\|_{\mathrm{p}}\right\|_{\mathrm{P}}, \quad w \in \mathrm{W}, \mathrm{p} \in \mathrm{P}, \\
\left|\mathrm{b}_{2}(v, \mathrm{q})\right| & \leq \overline{\mathrm{b}}_{2}\|v\|_{V}\left\|_{\mathrm{q}}\right\|_{\mathrm{Q}}, \quad v \in \mathrm{V}, \mathrm{q} \in \mathrm{Q}, \\
|\mathrm{c}(\mathrm{p}, \mathrm{q})| & \leq \overline{\mathrm{c}}\|\mathrm{p}\|_{\mathrm{P}}\|\mathrm{q}\|_{\mathrm{Q}}, \quad \mathrm{p} \in \mathrm{P}, \mathrm{q} \in \mathrm{Q},
\end{aligned}
$$

where $\bar{a}, \bar{b}_{1}, \bar{b}_{2}$ and $\bar{c}$ are continuity constants of the bilinear forms $a(\cdot, \cdot)$, $\mathrm{b}_{1}(\cdot, \cdot), \mathrm{b}_{2}(\cdot, \cdot)$ and $\mathrm{c}(\cdot, \cdot)$, respectively, and $\|\cdot\|_{\mathrm{V}},\|\cdot\|_{\mathrm{W}},\|\cdot\|_{\mathrm{P}}$ and $\|\cdot\|_{\mathrm{Q}}$ are induced inner products from the associated norms.

We define $\mathrm{U}_{\mathrm{W}} \subset \mathrm{W}$ and $\mathrm{U}_{\mathrm{V}} \subset \mathrm{V}$ as

$$
\begin{aligned}
& \mathrm{U}_{W}:=\left\{w \in W: b_{1}(w, p)=0, p \in P\right\}, \\
& \mathrm{U}_{\mathrm{V}}:=\left\{v \in \mathrm{V}: \mathrm{b}_{2}(v, \mathrm{q})=0, \mathrm{q} \in \mathrm{Q}\right\},
\end{aligned}
$$


and assume that

$$
\begin{aligned}
& \sup _{w \in \mathrm{U}_{W}} \frac{\mathrm{a}(v, w)}{\|w\|_{w}} \geq \alpha\|v\|_{V}, \quad v \in \mathrm{U}_{V}, \\
& \sup _{v \in \mathrm{U}_{V}} \mathrm{a}(v, w)>0, \quad w \in \mathrm{U}_{W} \backslash\{0\}, \\
& \sup _{w \in W} \frac{\mathrm{b}_{1}(w, p)}{\|w\|_{W}} \geq \beta_{1}\|p\|_{P}, \quad p \in P, \\
& \sup _{v \in V} \frac{\mathrm{b}_{2}(v, \mathrm{q})}{\|v\|_{V}} \geq \beta_{2}\|\mathrm{q}\|_{\mathrm{Q}}, \quad \mathrm{q} \in \mathrm{Q}
\end{aligned}
$$

hold for some constants $\alpha, \beta_{1}, \beta_{2}>0$, where the supremum is taken only over the non-trivial elements of the underlying sets. In order to show the existence and uniqueness of the problem (1), we need the following theorem, which was proved by Nicolaides [6] and Bernardi et al. [7].

Theorem 1 Let assumptions (2) and (3) be satisfied. Then for any $\mathrm{f} \in \mathrm{W}^{\prime}$ and $\mathrm{g} \in \mathrm{Q}^{\prime}$, there exists a unique solution $(\mathrm{u}, \mathrm{p}) \in \mathrm{V} \times \mathrm{P}$ to the saddle point problem of finding $(\mathrm{u}, \mathrm{p}) \in \mathrm{V} \times \mathrm{P}$ so that

$$
\begin{aligned}
\mathrm{a}(\mathrm{u}, w)+\mathrm{b}_{1}(w, p) & =\mathrm{f}(w), \quad w \in W \\
b_{2}(u, q) & =\mathrm{g}(\mathrm{q}), \quad \mathrm{q} \in \mathrm{Q},
\end{aligned}
$$

which satisfies the following stability estimates:

$$
\|u\|_{V} \leq \beta_{2}^{-1}\left(1+\alpha^{-1} \bar{a}\right)\|g\|_{Q^{\prime}}+\alpha^{-1}\|f\|_{W^{\prime}}, \quad\|p\|_{P} \leq \beta_{1}^{-1}\left(\|f\|_{W^{\prime}}+\bar{a}\|u\|_{V}\right) .
$$

The existence and uniqueness of the problem (1) is then established by the following theorem. Although we use a non-symmetric formulation in contrast to Ciarlet et al. [8], the proof of this theorem is similar [8, Theorem 3.2]. 
Theorem 2 Let assumptions (2) and (3) be satisfied, and

$$
\delta:=\beta_{1}^{-1} \beta_{2}^{-1} \overline{\mathrm{a}}\left(1+\alpha^{-1} \overline{\mathrm{a}}\right) \mathrm{t} \overline{\mathrm{c}}<1 .
$$

Then for any $\mathrm{f} \in \mathrm{V}^{\prime}$ and $\mathrm{g} \in \mathrm{Q}^{\prime}$, there exists a unique solution $(\mathrm{u}, \mathrm{p}) \in \mathrm{V} \times \mathrm{P}$ to the saddle point problem (1) satisfying the following stability estimates:

$$
\|p\|_{P} \leq \frac{1}{1-\delta}\|\tilde{p}\|_{P}, \quad\|u\|_{V} \leq\|\tilde{u}\|_{V}+\frac{\beta_{2}\left(1+\alpha^{-1} \bar{a}\right) t \bar{c}}{1-\delta}\|\tilde{p}\|_{P}
$$

where $(\tilde{\mathrm{u}}, \tilde{\mathrm{p}})$ is the solution to (4) and satisfies the bounds

$$
\|\tilde{u}\|_{V} \leq \beta_{2}^{-1}\left(1+\alpha^{-1} \bar{a}\right)\|g\|_{Q^{\prime}}+\alpha^{-1}\|f\|_{W^{\prime}}, \quad\|\tilde{p}\|_{P} \leq \beta_{1}^{-1}\left(\|f\|_{W^{\prime}}+\bar{a}\|\tilde{u}\|_{V}\right) .
$$

Proof: Letting $p_{0}=0 \in P$, we define a sequence $\left\{\left(u_{n}, p_{n}\right)\right\}$ for $n \in \mathbb{N}$ by

$$
\begin{aligned}
\mathrm{a}\left(\mathrm{u}_{\mathrm{n}+1}, w\right)+b_{1}\left(w, p_{n+1}\right) & =\mathrm{f}(w), \quad w \in W \\
b_{2}\left(u_{n+1}, q\right) & =g(q)+\mathrm{tc}\left(p_{n}, q\right), \quad q \in Q .
\end{aligned}
$$

The sequence is well-defined from Theorem 1 , and for $n \in \mathbb{N}$ we have

$$
\begin{aligned}
\mathrm{a}\left(\mathrm{u}_{\mathrm{n}+1}-\mathrm{u}_{\mathrm{n}}, w\right)+\mathrm{b}_{1}\left(w, p_{n+1}-p_{n}\right) & =0, \quad w \in W \\
b_{2}\left(u_{n+1}-u_{n}, q\right) & =\operatorname{tc}\left(p_{n}-p_{n-1}, q\right), \quad q \in Q .
\end{aligned}
$$

Theorem 1 yields the existence and uniqueness of the solution of (9) with the estimates

$$
\begin{aligned}
\left\|u_{n+1}-u_{n}\right\|_{V} & \leq \beta_{2}^{-1}\left(1+\alpha^{-1} \bar{a}\right) t \bar{c}\left\|p_{n}-p_{n-1}\right\|_{P} \\
\left\|p_{n+1}-p_{n}\right\|_{P} & \leq \beta_{1}^{-1} \bar{a}\left\|u_{n+1}-u_{n}\right\|_{V}
\end{aligned}
$$

and hence

$$
\left\|p_{n+1}-p_{n}\right\|_{P} \leq \beta_{1}^{-1} \beta_{2}^{-1} \bar{a}\left(1+\alpha^{-1} \bar{a}\right) t \bar{c}\left\|p_{n}-p_{n-1}\right\|_{P} \leq \delta^{n}\left\|p_{1}\right\|_{P} .
$$


Now taking $n \in \mathbb{N}$ and an integer $\boldsymbol{m}>\mathfrak{n}$, we have

$$
\left\|p_{m}-p_{n}\right\|_{P} \leq \sum_{i=n}^{m-1}\left\|p_{i+1}-p_{i}\right\|_{P} \leq \sum_{i=n}^{m-1} \delta^{i}\left\|p_{1}\right\|_{P} \leq \frac{\delta^{n}}{1-\delta}\left\|p_{1}\right\|_{P},
$$

which shows that $\left\{p_{n}\right\}$ is a Cauchy sequence, and so converges to a $p \in P$. The stability estimate for $p$ is obtained by taking $\mathfrak{n}=0$ in (12). Using the first inequality of (10) and the estimate (11), the sequence $\left\{\boldsymbol{u}_{n}\right\}$ is shown to be a Cauchy sequence, and stability estimate for $u$ is obtained similarly as for $p$. The uniqueness and other details is to be worked out as in the work of Ciarlet et al. [8, Theorem 3.2].

Our primary concern is to find a robust approximation scheme based on linear finite elements and simplicial triangulation for the nearly incompressible elastic problem. In particular, we are interested in a mixed scheme where the pressure variable is eliminated and obtain a formulation based only on the displacement variable.

Let $\Omega \subset \mathbb{R}^{\mathrm{d}}$ with $\mathrm{d} \in\{2,3\}, \mathrm{L}^{2}(\Omega)$ be the space of square-integrable functions defined on $\Omega$ with the inner product and norm being denoted by $(\cdot, \cdot)_{0}$ and $\|\cdot\|_{0}$, respectively, and $\mathrm{L}_{0}^{2}(\Omega):=\left\{\mathrm{p} \in \mathrm{L}^{2}(\Omega): \int_{\Omega} \mathrm{p} d x=0\right\}$. The space $H_{0}^{1}(\Omega)$ consists of functions in $H^{1}(\Omega)$ which vanish on the boundary in the sense of traces. To write the weak or variational formulation of the boundary value problem, we introduce the space $\mathbf{V}:=\left[\mathrm{H}_{0}^{1}(\Omega)\right]^{\mathrm{d}}$ of displacements with inner product $(\cdot, \cdot)_{1}$ and norm $\|\cdot\|_{1}$ defined in the standard way; that is, $(\boldsymbol{u}, \boldsymbol{v})_{1}:=\sum_{i=1}^{\mathrm{d}}\left(u_{i}, v_{i}\right)_{1}$, with the norm being induced by this inner product. A mixed formulation of the linear elastic problem is given by introducing pressure as an extra variable leading to penalized Stokes equations [4]. Defining $p:=\lambda \operatorname{div} \mathbf{u}$, a mixed variational formulation of linear elastic problem is: given $\ell \in\left[\mathrm{L}^{2}(\Omega)\right]^{\mathrm{d}}$, find $(\mathbf{u}, p) \in \mathbf{V} \times \mathrm{L}_{0}^{2}(\Omega)$ such that

$$
\begin{aligned}
\mathrm{A}(\mathbf{u}, \boldsymbol{v})+\mathrm{B}(\boldsymbol{v}, p) & =\ell(\boldsymbol{v}), \quad \boldsymbol{v} \in \mathbf{V}, \\
\mathrm{B}(\mathbf{u}, \mathrm{q})-\frac{1}{\lambda} \mathrm{C}(\mathrm{p}, \mathrm{q}) & =0, \quad \mathrm{q} \in \mathrm{L}_{0}^{2}(\Omega),
\end{aligned}
$$


where $A(\boldsymbol{u}, \boldsymbol{v}):=2 \mu \int_{\Omega} \varepsilon(u): \varepsilon(v) d x, B(v, q):=\int_{\Omega} \operatorname{div} \boldsymbol{v} q \mathrm{dx}, \mathrm{C}(\mathrm{p}, \mathrm{q}):=$ $\int_{\Omega} p q d x$ and $\ell(\boldsymbol{v}):=\int_{\Omega} f \cdot v d x$. Here, $\lambda$ and $\mu$ are Lamé parameters, $\varepsilon(u)$ is the strain of the displacement defined as $\mathfrak{\varepsilon}(\mathbf{u})=\frac{1}{2}\left(\nabla \mathbf{u}+[\nabla \mathbf{u}]^{\top}\right)$ and $\mathfrak{f}$ is the prescribed body force. The existence and uniqueness of the solution of the problem (13) is shown by a standard theory of saddle point problems [3, 4].

\section{Finite element discretization}

We consider a quasi-uniform triangulation $\mathcal{T}_{h}$ of the polygonal or polyhedral domain $\Omega$, where $\mathcal{T}_{h}$ consists of simplices, either triangles or tetrahedra. Making use of the standard linear finite element space $S_{h}$ defined on the triangulation $\mathcal{T}_{h}$,

$$
S_{\mathrm{h}}:=\left\{v \in \mathrm{H}^{1}(\Omega): v_{\left.\right|_{\mathrm{T}}} \in \mathcal{P}_{1}(\mathrm{~T}), \mathrm{T} \in \mathcal{T}_{\mathrm{h}}\right\},
$$

and the space of bubble functions

$$
\mathrm{B}_{\mathrm{h}}:=\left\{\mathrm{b}_{\mathrm{T}} \in \mathrm{H}^{1}(\mathrm{~T}): \mathrm{b}_{\mathrm{T}_{\mathrm{\partial} \mathrm{T}}}=0 \text { and } \int_{\mathrm{T}} \mathrm{b}_{\mathrm{T}} \mathrm{d} \mathrm{x}>0, \mathrm{~T} \in \mathcal{T}_{\mathrm{h}}\right\},
$$

we introduce our finite element space for the displacement as $\mathbf{V}_{\mathrm{h}}=\left(\mathrm{S}_{\mathrm{h}} \oplus\right.$ $\left.B_{h}\right)^{d} \cap \mathbf{V}$. The bubble function on an element $T$ is most often defined as $\mathrm{b}_{\mathrm{T}}(\mathrm{x})=\mathrm{c}_{\mathrm{b}} \prod_{i=1}^{\mathrm{d}+1} \lambda_{\mathrm{T}^{\mathrm{i}}}(\mathrm{x})$, where $\lambda_{\mathrm{T}^{\mathrm{i}}}(\mathrm{x})$ are the barycentric coordinates of the element $T$ associated with vertices $x_{T^{i}}$ of $T, i=1, \ldots, d+1$. The constant $c_{b}$ is computed in such a way that the value of the bubble function at the barycenter of $\mathrm{T}$ is one. Let $\mathrm{N}$ be the number of nodes in the finite element mesh, and $\left\{\phi_{1}, \ldots, \phi_{N}\right\}$ be the finite element basis of $S_{h}$. Starting with the basis of $S_{h}$, we construct a dual space $Q_{h}$ spanned by the basis $\left\{\mu_{1}, \ldots, \mu_{N}\right\}$ so that the basis functions of $S_{h}$ and $Q_{h}$ satisfy biorthogonality:

$$
\int_{\Omega} \mu_{i} \phi_{j} d x=c_{j} \delta_{i j}, \quad c_{j} \neq 0, \quad 1 \leq i, j \leq N,
$$


where $\delta_{i j}$ is the Kronecker symbol, and $c_{j}$ a scaling factor, which is chosen so that $\int_{T} \mu_{i} d x=\int_{T} \phi_{i} d x$. Hence, the sets of basis functions of $S_{h}$ and $Q_{h}$ form a biorthogonal system. The basis functions of $Q_{h}$ is constructed locally on the reference element $\hat{T}_{\text {so }}$ so that basis functions of $S_{h}$ and $Q_{h}$ have the same support [5]. We also need a subspace of $S_{h}$ and a subspace of $Q_{h}$ having zero average on $\Omega$ defined as

$$
S_{h}^{0}:=\left\{v_{h} \in S_{h}: \int_{\Omega} v_{h} d x=0\right\}, \quad Q_{h}^{0}:=\left\{q_{h} \in Q_{h}: \int_{\Omega} q_{h} d x=0\right\} .
$$

The first equation in (13) is discretized using a Galerkin formulation, and the second equation is discretized using a Petrov-Galerkin formulation. The Petrov-Galerkin formulation is chosen so that the pressure solution is taken from $S_{h}$, whereas the test functions are taken from $Q_{h}$. Hence the discrete formulation of variational equation (13) is written as: find $\left(\mathbf{u}_{\mathrm{h}}, \mathbf{p}_{\mathrm{h}}\right) \in \mathbf{V}_{\mathrm{h}} \times \mathrm{S}_{\mathrm{h}}^{0}$ such that

$$
\begin{aligned}
\mathrm{A}\left(\boldsymbol{u}_{\mathrm{h}}, \boldsymbol{v}_{\mathrm{h}}\right)+\mathrm{B}_{1}\left(\boldsymbol{v}_{\mathrm{h}}, \mathrm{p}_{\mathrm{h}}\right) & =\ell\left(\boldsymbol{v}_{\mathrm{h}}\right), \quad \boldsymbol{v}_{\mathrm{h}} \in \mathbf{V}_{\mathrm{h}}, \\
\mathrm{B}_{2}\left(\mathbf{u}_{\mathrm{h}}, \mathrm{q}_{\mathrm{h}}\right)-\frac{1}{\lambda} \mathrm{C}\left(\mathrm{p}_{\mathrm{h}}, \mathrm{q}_{\mathrm{h}}\right) & =0, \quad \mathrm{q}_{\mathrm{h}} \in \mathrm{Q}_{\mathrm{h}}^{\mathrm{O}},
\end{aligned}
$$

where the bilinear forms $B_{1}(\cdot, \cdot): \mathbf{V}_{h} \times S_{h}^{0} \rightarrow \mathbb{R}$ and $B_{2}(\cdot, \cdot): V_{h} \times Q_{h}^{0} \rightarrow \mathbb{R}$ have different domains of definition but are defined exactly as the bilinear form $B(\cdot, \cdot)$ in (13). The goal of choosing the Petrov-Galerkin formulation for the pressure is to obtain a diagonal matrix corresponding to the bilinear form $C(\cdot, \cdot)$ so that the degree of freedom corresponding to the pressure variable is eliminated easily.

We show the existence and uniqueness of the solution of the mixed formulation (15) using Theorem 2.

The continuity of the bilinear form $A(\cdot, \cdot)$ on $\mathbf{V}_{h} \times \mathbf{V}_{h}$, of $B_{1}(\cdot, \cdot)$ on $\mathbf{V}_{h} \times$ $S_{h}^{0}$, and $B_{2}(\cdot, \cdot)$ on $V_{h} \times Q_{h}^{0}$ and of $C(\cdot, \cdot)$ on $S_{h}^{0} \times Q_{h}^{0}$ is straightforward. By using the Korn's inequality, it is standard that the ellipticity of the bilinear 
form $A(\cdot, \cdot)$ holds on $\mathbf{V}_{h} \times \mathbf{V}_{h}$. It remains to show that the uniform inf-sup condition holds for the bilinear form $B_{1}(\cdot, \cdot)$ on $\mathbf{V}_{h} \times S_{h}^{0}$, and for the bilinear form $B_{2}(\cdot, \cdot)$ on $V_{h} \times Q_{h}^{0}$. As the bilinear form $B_{1}(\cdot, \cdot)$ on $V_{h} \times S_{h}^{0}$ satisfies the inf-sup condition uniformly with respect to the mesh size [9], we turn our attention to prove a uniform inf-sup condition for $B_{2}(\cdot, \cdot)$ on $\mathbf{V}_{h} \times Q_{h}^{0}$. That means we have to show the existence of a constant $\beta>0$ independent of the mesh-size such that

$$
\sup _{\boldsymbol{v}_{\mathrm{h}} \in \mathbf{V}_{\mathrm{h}}} \frac{\mathrm{B}_{2}\left(\boldsymbol{v}_{\mathrm{h}}, \mathrm{q}_{\mathrm{h}}\right)}{\left\|\boldsymbol{v}_{\mathrm{h}}\right\|_{1}} \geq \beta\left\|\mathrm{q}_{\mathrm{h}}\right\|_{0}, \quad \mathrm{q}_{\mathrm{h}} \in \mathrm{Q}_{\mathrm{h}}^{0}
$$

To this end, we define an operator $I_{h}: Q_{h}^{0} \rightarrow S_{h}^{0}$ mapping every element $\mu_{h}=\sum_{i=1}^{n} c_{i} \mu_{i}$ of $Q_{h}^{0}$ to the element $\phi_{h}=\sum_{i=1}^{n} c_{i} \phi_{i}$ of $S_{h}^{0}$. Using that $\int_{T} \phi_{i} d x=\int_{T} \mu_{i} d x, 1 \leq i \leq d+1$, we have $\int_{T}^{i=1} \phi_{h} d x=\int_{T} I_{h} \phi_{h} d x$, and hence the operator is well-defined.

For the reference triangle or reference tetrahedron, using Gauss divergence theorem, we have the following identity for any bubble function $b_{T} \in B_{h}$

$$
\int_{T} \nabla b_{T} \mu_{i} d x=(d+2) \int_{T} \nabla b_{T} \phi_{i} d x .
$$

Using (17), we show the following properties of the operator $I_{h}$.

Lemma 3 Given $\boldsymbol{v}_{\mathrm{h}} \in \mathbf{V}_{\mathrm{h}}$, we construct an element $\tilde{\boldsymbol{v}}_{\mathrm{h}} \in \mathbf{V}_{\mathrm{h}}$ so that there exists a constant $\mathrm{c}>0$ with

$$
\left\|\tilde{\boldsymbol{v}}_{\mathrm{h}}\right\|_{1} \leq \mathrm{c}\left\|\boldsymbol{v}_{\mathrm{h}}\right\|_{1}, \quad \mathrm{~B}_{2}\left(\tilde{\boldsymbol{v}}_{\mathrm{h}}, \mathrm{q}_{\mathrm{h}}\right)=\mathrm{B}_{2}\left(\boldsymbol{v}_{\mathrm{h}}, \mathrm{I}_{\mathrm{h}} \mathrm{q}_{\mathrm{h}}\right), \quad \mathrm{q}_{\mathrm{h}} \in \mathrm{Q}_{\mathrm{h}}^{\mathrm{O}},
$$

and there exist two constants $\mathrm{c}_{1}>0$ and $\mathrm{c}_{2}>0$ with

$$
c_{1}\left\|\mathrm{q}_{h}\right\|_{0} \leq\left\|\mathrm{I}_{\mathrm{h}} \mathrm{q}_{\mathrm{h}}\right\|_{0} \leq \mathrm{c}_{2}\left\|\mathrm{q}_{\mathrm{h}}\right\|_{0} .
$$


Proof: We start with

$B_{2}\left(v_{h}, q_{h}-I_{h} q_{h}\right)=\int_{\Omega} \operatorname{div} v_{h}\left(q_{h}-I_{h} q_{h}\right) d x=\sum_{T \in \mathcal{T}_{h}} \int_{T} \operatorname{div} v_{h}\left(q_{h}-I_{h} q_{h}\right) d x$

for $\boldsymbol{v}_{\mathrm{h}} \in \mathbf{V}_{\mathrm{h}}$ and $\mathrm{q}_{\mathrm{h}} \in \mathrm{Q}_{\mathrm{h}}^{0}$. Let $\boldsymbol{v}_{\mathrm{h}}=\mathbf{s}_{\mathrm{h}}+\mathbf{b}_{\mathrm{h}}$ with $\mathbf{s}_{\mathrm{h}} \in \mathrm{S}_{\mathrm{h}}^{\mathrm{d}}$, and $\mathbf{b}_{\mathrm{h}} \in \mathrm{B}_{\mathrm{h}}^{\mathrm{d}}$. Then

$B_{2}\left(v_{h}, q_{h}-I_{h} q_{h}\right)=\sum_{T \in \mathcal{T}_{h}} \int_{T} \operatorname{div} s_{h}\left(q_{h}-I_{h} q_{h}\right) d x+\int_{T} \operatorname{div} b_{h}\left(q_{h}-I_{h} q_{h}\right) d x$.

Using that div $\mathbf{s}_{\mathrm{h}}$ is constant in each element $\mathrm{T}$, the first integral in the right side of the above equation is zero, and hence

$$
B_{2}\left(v_{h}, q_{h}-I_{h} q_{h}\right)=B_{2}\left(b_{h}, q_{h}-I_{h} q_{h}\right) .
$$

As $\mathrm{q}_{\mathrm{h}} \in \mathrm{Q}_{\mathrm{h}}^{0}$, we write $\mathrm{q}_{\mathrm{h}}=\sum_{i=1}^{\mathrm{N}} \mathrm{q}_{\mathrm{i}} \mu_{\mathrm{i}}$ and obtain

$$
\begin{aligned}
\mathrm{B}_{2}\left(\mathbf{b}_{\mathrm{h}}, \mathbf{q}_{\mathrm{h}}\right) & =\sum_{i=1}^{N} \mathrm{q}_{\mathrm{i}} \int_{S_{\mathrm{i}}} \operatorname{div} \mathbf{b}_{\mathrm{h}} \mu_{\mathrm{i}} \mathrm{d} x, \\
\text { and } \quad \mathrm{B}_{2}\left(\mathbf{b}_{\mathrm{h}}, \mathrm{I}_{\mathrm{h}} \mathrm{q}_{\mathrm{h}}\right) & =\sum_{i=1}^{N} \mathrm{q}_{\mathrm{i}} \int_{S_{\mathrm{i}}} \operatorname{div} \mathbf{b}_{\mathrm{h}} \phi_{\mathrm{i}} \mathrm{d} x,
\end{aligned}
$$

where $S_{i}$ denotes the support of $\phi_{i}$ or $\mu_{i}$. Since $\boldsymbol{b}_{h}$ belongs to the space of bubble functions, restricted to an element $T$, it is written as $\mathbf{b}_{h}=\mathbf{a}_{\mathrm{T}} \mathbf{b}_{\mathrm{T}}$ for some constant vector $\boldsymbol{a}_{\mathrm{T}}$. Now we decompose the integrals inside both sums into each element

$$
\begin{aligned}
\mathrm{B}_{2}\left(\mathbf{b}_{\mathrm{h}}, \mathrm{q}_{\mathrm{h}}\right) & =\sum_{i=1}^{\mathrm{N}} \mathrm{q}_{\mathrm{i}} \sum_{\mathrm{T} \subset \mathrm{S}_{i}} \boldsymbol{a}_{\mathrm{T}} \cdot \int_{\mathrm{T}} \nabla \mathrm{b}_{\mathrm{T}} \mu_{\mathrm{i}} \mathrm{d} x, \\
\mathrm{~B}_{2}\left(\mathbf{b}_{\mathrm{h}}, \mathrm{I}_{\mathrm{h}} \mathrm{q}_{\mathrm{h}}\right) & =\sum_{i=1}^{N} \mathrm{q}_{i} \sum_{\mathrm{T} \subset \mathrm{S}_{\mathrm{i}}} \boldsymbol{a}_{\mathrm{T}} \cdot \int_{\mathrm{T}} \nabla \mathrm{b}_{\mathrm{T}} \phi_{i} \mathrm{~d} x,
\end{aligned}
$$


and use the property of a bubble function (17) to get

$$
B_{2}\left(b_{h}, q_{h}\right)=(d+2) B_{2}\left(b_{h}, I_{h} q_{h}\right) .
$$

Hence defining $\tilde{\boldsymbol{v}}_{\mathrm{h}}:=\mathbf{s}_{\mathrm{h}}+\frac{1}{\mathrm{~d}+2} \mathbf{b}_{\mathrm{h}}$, and noting that $\mathbf{s}_{\mathrm{h}}$ and $\mathbf{b}_{\mathrm{h}}$ are linearly independent the first condition (18) is proved. The second condition (19) follows by using the fact that $\left\|I_{h} q_{h}\right\|_{0}^{2},\left\|q_{h}\right\|_{0}^{2}$ and $\sum_{i=1}^{N} q_{i}^{2} h_{i}^{2}$ are equivalent, where $h_{i}$ is the local mesh-size at the ith node of $\mathcal{T}_{h}$.

A consequence of the above lemma is the following theorem.

Theorem 4 The finite element pair $\left(\mathbf{V}_{\mathrm{h}}, \mathrm{Q}_{\mathrm{h}}^{0}\right)$ satisfies the inf-sup condition (16).

Proof: Let $\mathrm{q}_{h} \in \mathrm{Q}_{h}^{0}$, and $\mathrm{I}_{h} \mathrm{q}_{\mathrm{h}} \in \mathrm{S}_{\mathrm{h}}^{0}$. Since the pair $\left(\mathbf{V}_{\mathrm{h}}, \mathrm{S}_{\mathrm{h}}^{0}\right)$ satisfies the inf-sup condition, we can find an element $\boldsymbol{v}_{\mathrm{h}} \in \mathbf{V}_{\mathrm{h}}$ satisfying

$$
B_{2}\left(v_{h}, I_{h} q_{h}\right) \geq c\left\|I_{h} q_{h}\right\|_{0}^{2} \text { and }\left\|v_{h}\right\|_{1} \leq c\left\|I_{h} q_{h}\right\|_{0} .
$$

Hence, using the properties (18) and (19) of the interpolation operator $I_{h}$, we can find an element $\tilde{\boldsymbol{v}}_{\mathrm{h}} \in \mathbf{V}_{\mathrm{h}}$ with

$$
B_{2}\left(\tilde{\boldsymbol{v}}_{h}, \mathrm{q}_{h}\right)=\mathrm{B}_{2}\left(\boldsymbol{v}_{\mathrm{h}}, \mathrm{I}_{\mathrm{h}} \mathrm{q}_{\mathrm{h}}\right) \geq \mathrm{c}\left\|\mathrm{I}_{\mathrm{h}} \mathrm{q}_{\mathrm{h}}\right\|_{0}^{2} \geq \mathrm{c}\left\|\mathrm{q}_{\mathrm{h}}\right\|_{0}^{2},
$$

and

$$
\left\|\tilde{\boldsymbol{v}}_{\mathrm{h}}\right\|_{1} \leq \mathrm{c}\left\|\boldsymbol{v}_{\mathrm{h}}\right\|_{1} \leq \mathrm{c}\left\|\mathrm{I}_{\mathrm{h}} \mathrm{q}_{\mathrm{h}}\right\|_{0} \leq \mathrm{c}\left\|\mathrm{q}_{\mathrm{h}}\right\|_{0}
$$

Thanks to Theorems 2 and 4, the following corollary holds $[6,7,3,10]$.

Corollary 5 The discrete problem (15) has exactly one solution $\left(\mathbf{u}_{\mathrm{h}}, \mathbf{p}_{\mathrm{h}}\right) \in$ $\mathbf{V}_{\mathrm{h}} \times \mathrm{S}_{\mathrm{h}}^{0}$, and there exists a constant $\mathrm{c}$ independent of Lamé parameter $\lambda$ such that

$$
\left\|\mathbf{u}_{\mathrm{h}}\right\|_{1}+\left\|p_{h}\right\|_{0} \leq \mathbf{c}\|\mathbf{f}\|_{0}
$$


Furthermore, if $(\mathbf{u}, \mathbf{p})$ is the solution to the problem (13), we have the following error estimate uniform with respect to $\lambda$ :

$$
\left\|\mathbf{u}-\mathbf{u}_{\mathrm{h}}\right\|_{1}+\left\|p-p_{\mathrm{h}}\right\|_{0} \leq \mathrm{c}_{1} \inf _{\boldsymbol{v}_{\mathrm{h}} \in \mathbf{V}_{\mathrm{h}}}\left\|\mathbf{u}-\boldsymbol{v}_{\mathrm{h}}\right\|_{1}+\mathrm{c}_{2} \inf _{\mathrm{q}_{\mathrm{h}} \in \mathrm{S}_{\mathrm{h}}^{0}}\left\|p-\mathrm{q}_{\mathrm{h}}\right\|_{0},
$$

where the constants $\mathrm{c}_{1}$ and $\mathrm{c}_{2}$ are independent of the mesh size.

Using the standard approximation properties of the spaces $\mathbf{V}_{\mathrm{h}}$ and $S_{h}^{0}$, we see that the approximation to the displacement converges to the exact solution with $\mathrm{O}(\mathrm{h})$ in $\mathrm{H}^{1}$-norm.

\section{$3 \quad$ Numerical results}

This section illustrates the performance of the formulation discussed in the preceding sections in a numerical example [11] showing a comparison of different formulations using $\mathrm{L}^{2}$ - and $\mathrm{H}^{1}$-norms. Here, the exact solution $\mathbf{u}=\left(\mathfrak{u}_{1}, \mathfrak{u}_{2}\right)$ is

$$
\begin{aligned}
& u_{1}(x, y):=\sin (2 \pi y)(-1+\cos (2 \pi x))+\frac{\sin (\pi x) \sin (\pi y)}{1+\lambda}, \\
& u_{2}(x, y):=\sin (2 \pi x)(1-\cos (2 \pi y))+\frac{\sin (\pi x) \sin (\pi y)}{1+\lambda}
\end{aligned}
$$

with $\lambda=2499666.644443238$ and $\mu=500.0333355557037$ (the corresponding Poisson ratio and Young's modulus are $v=0.4999$ and $E=1500$ ) so that a nearly incompressible response is obtained. We compute the solution by taking $\Omega$ as a unit square, where the right hand side and the Dirichlet boundary conditions are computed by using the exact solution. We have shown the discretization errors with respect to the number of elements in Figure 1. As is seen from Figure 1, the standard approach locks completely, whereas we get very good numerical approximations with our approach and mini-element. However, our formulation is more efficient as we reduce the problem to the displacement-based formulation by inverting a diagonal matrix. 

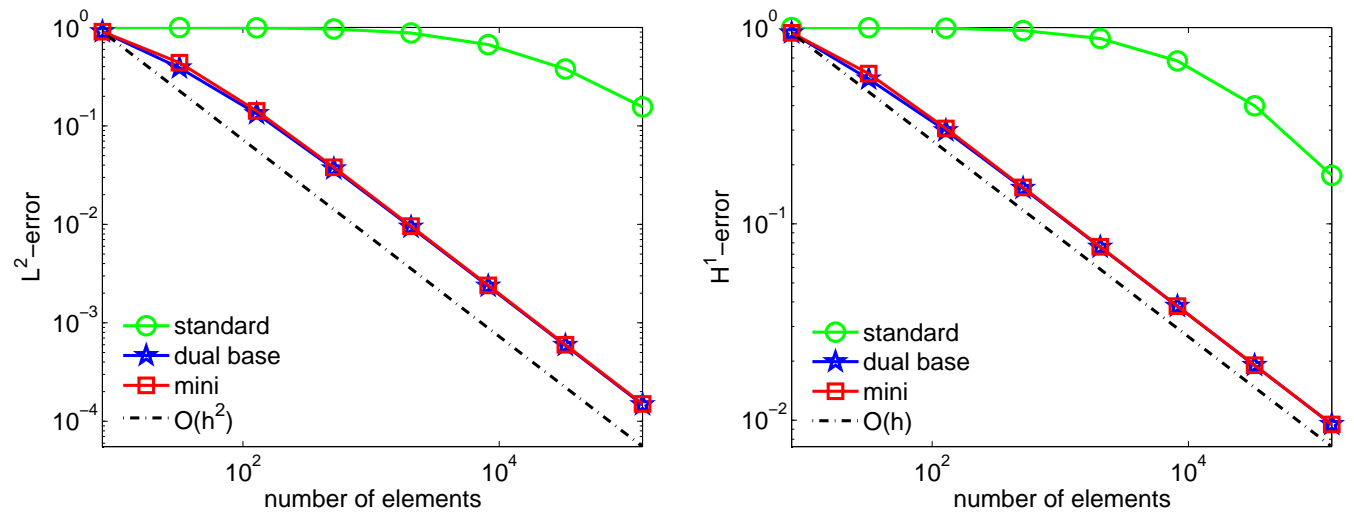

FiguRE 1: Error plot versus number of elements, $\mathrm{L}^{2}$-norm (left) and $\mathrm{H}^{1}$-norm (right).

\section{References}

[1] Babuska, I. and Suri, M. (1992). Locking effects in the finite element approximation of elasticity problems. Numerische Mathematik, 62, 439-463. doi:10.1007/BF01396238 C325

[2] Babuška, I. and Suri, M. (1992). On locking and robustness in the finite element method. SIAM Journal on Numerical Analysis, 29, 1261-1293. doi:10.1137/0729075 C325

[3] Brezzi, F. and Fortin, M. (1991). Mixed and hybrid finite element methods. Springer-Verlag, New York. C325, C326, C330, C334

[4] Braess, D. (1996). Stability of saddle point problems with penalty. Mathematical Modelling and Numerical Analysis, 30, 731-742. C325, C326, C329, C330

[5] Lamichhane, B. (2008). Inf-sup stable finite element pairs based on dual meshes and bases for nearly incompressible elasticity. IMA 
Journal of Numerical Analysis. doi:10.1093/imanum/drn013 C325, C331

[6] Nicolaides, R. (1982). Existence, uniqueness and approximation for generalized saddle point problems. SIAM Journal on Numerical Analysis, 19, 349-357. doi:10.1137/0719021 C325, C326, C327, C334

[7] Bernardi, C., Canuto, C. and Maday, Y. (1988). Generalized inf-sup conditions for Chebyshev spectral approximation of the Stokes problem. SIAM Journal on Numerical Analysis, 25, 1237-1271. doi:10.1137/0725070 C325, C326, C327, C334

[8] Ciarlet, P. J., Huang, J. and Zou, J. (2003). Some observations on generalized saddle-point problems. SIAM Journal on Matrix Analysis and Applications, 25, 224-236. doi:10.1137/S0895479802410827 C325, C326, C327, C329

[9] Arnold, D. N., Brezzi, F. and Fortin, M. (1984). A stable finite element for the Stokes equations. Calcolo, 21, 337-344. doi:10.1007/BF02576171 C325, C332

[10] Braess, D. (2001). Finite Elements. Theory, fast solver, and applications in solid mechanics. Cambridge University Press, Second Edition. C334

[11] Brenner, S. (1993). A nonconforming mixed multigrid method for the pure displacement problem in planar linear elasticity. SIAM Journal on Numerical Analysis, 30, 116-135. doi:10.1137/0730006 C335 


\section{Author address}

1. Bishnu P. Lamichhane, Centre for Mathematics and its Applications, Mathematical Sciences Institute, Australian National University, ACT 0200, Canberra, Australia.

mailto:Bishnu.Lamichhane@maths . anu .edu .au 